\title{
Calculation and Interpretation of Ground Penetrating Radar for Temperature and Relative Water Content of Seasonal Permafrost in Qinghai-Tibet Plateau
}

\author{
Qing Wang ${ }^{1}$ and Yupeng Shen ${ }^{2, *}$ \\ 1 School of Information and Communication Engineering, Beijing Information Science and Technology \\ University, 100192 Beijing, China \\ 2 School of Civil Engineering, Beijing Jiaotong University, 100044 Beijing, China \\ * Correspondence: ypshen@bjtu.edu.cn; Tel.: +86-1381-087-3345
}

Received: 1 May 2019; Accepted: 25 June 2019; Published: 27 June 2019

\begin{abstract}
Due to the seasonal permafrost thawing, the Qinghai-Tibet Highway has a depression and instability of the roadbed. In order to obtain the ablation interface and water content characteristics of seasonal permafrost areas, non-destructive ground penetrating radars using electromagnetic wave detection methods are widely used. Regarding the imaging of the ablation interface in permafrost regions, this paper proposes a high-precision procedure for seasonal permafrost media using waveform difference analysis, electromagnetic wave attenuation attribute calculation and relative wave impedance conversion. It improves the resolution and division accuracy of the imaging. In addition, the study demonstrates a method to calculate the temperature and water content of the ablation zone by mining attenuation attribute, relative wave impedance attribute, absolute instantaneous amplitude attribute and the weighted average frequency attribute parameters under the constraints of the measured data. This method has high accuracy and high efficiency and can be used in the rapid calculation of temperature and water content of seasonal permafrost on the Qinghai-Tibet Highway.
\end{abstract}

Keywords: GPR; seasonal permafrost; electromagnetic wave attribute; relative water content

\section{Introduction}

Various rocks and soils with temperatures below $0{ }^{\circ} \mathrm{C}$ and containing ice are often referred to as frozen soils. According to the time when the soil is in a state of continuous freezing, the frozen soil is generally divided into short-term frozen soil (hours to half-months), seasonal permafrost (half months to several months), and permafrost (several years to tens of thousands of years). China's permafrost is mainly distributed in the Qinghai-Tibet Plateau, the northeastern Daxing, Xiaoxing'anling, Songnen Plain and some high mountains in the west, with a total area of about $2.07 \times 106 \mathrm{~km}^{2}$, accounting for $21.5 \%$ of China's land area. Permafrost in the Qinghai-Tibet Plateau is the representative of high-altitude permafrost in the low latitudes of the world, with a distribution area of about $1.5 \times$ $106 \mathrm{~km}^{2}$, accounting for $70 \%$ of the total permafrost area in China [1].

Permafrost is extremely sensitive to temperature changes, especially high-altitude permafrost, and slight changes in temperature have a significant impact on the temperature and stability of Permafrost. [2,3]. According to the temperature data of the central hole along the Qinghai-Tibet Highway, a thawing nucleus with a thickness of 2-7 $\mathrm{m}$ has been formed under the Qinghai-Tibet Highway, and tens of kilometers of a "thawing channel" has been formed under the roadbed along the longitudinal direction of the road. Most of the thawing nucleus stores water for many years, resulting in strong uneven subsidence and road surface damage [4,5]. Therefore, detecting the interface of seasonal permafrost thawing, the formation temperature and the water content in the soil are of great significance for judging the stability and safety of the road project. In addition, it also provides data for global warming research. 
As the seasons and temperature change, the dielectric constant and the conductivity of soil in seasonal permafrost soils change significantly. The dielectric constant of the seasonal permafrost soil area can vary from 3 to 30. These changes will be directly reflected in the geophysical wave field [6]. The ground penetrating radar (GPR) using electromagnetic wave detection method has been widely used since the 1990s because of its advantages of being fast, of high efficiency and non-destructive [7]. Arcone et al. [8,9] of the US Army Engineering Cold Research and Engineering Laboratory (CRREL) successfully detected groundwater below the frozen soil in permafrost and rock masses that exist within the permanent frozen layer in Alaska using GPR of $100 \mathrm{MHz}$ and $50 \mathrm{MHz}$. De Pascale et al. (2008) used a combination of GPR and capacitively coupled resistivity profilometry to examine the distribution of ice bodies in multi-year frozen layers on several sections of Richards Island in the western Canadian Arctic. Xiao and Liu [10] used dual-frequency (100 MHz and $400 \mathrm{MHz}$ ) ground penetrating radar data to study the important layered interfaces such as the seasonal active layer and the top surface of the frozen soil. These studies mainly focus on two aspects: 1) the size, shape and distribution of ice bodies in the permafrost; 2) the layered structure of the thermal state. In the study of permafrost soil's water content study using GPR data, scholars have proposed many theoretical and empirical model formulas. The more famous formulas are: Topp formula, Malicki formula, Roth formula and Alharathi formula [11,12]. Du and Sperl proposed a method for measuring soil water content using GPR common offset data [13]. Huisman [14] et al. used the WARR method to apply $225 \mathrm{MHz}$ and $450 \mathrm{MHz}$ antennas respectively to determine the water content of the soil based on the slope of the ground wave. Shen et al. [15] improved the Topp formula using the GPR instantaneous quality factor attribute to study the relative water content of frozen soil. However, these studies also have the following problems. Firstly, in the study of seasonal permafrost soil in the Qinghai-Tibet Plateau, the division of different temperature layers and thawing layers is not elaborate enough, and only the in-phase reflection of GPR data is utilized. No more GPR data attributes have been analyzed. Secondly, there are few studies on using the GPR data to calculate the water content in the frozen soil area. The method of using only a single attribute for water content calculation is not precise enough and lacks constraints.

In order to solve these problems, this study comprehensively analyzed the characteristics of GPR data attributes in seasonal permafrost soil areas. Accurate imaging and stratigraphic division of seasonal frozen soil on the Qinghai-Tibet Plateau are carried out in terms of waveform variation, wavefield attenuation and wave impedance variation. In addition, under the constraint of water content and temperature data, the water content measurement model of frozen soil area was reconstructed by comprehensively utilizing various GPR data sensitive attributes. The water content and formation temperature of the measurement area were calculated.

\section{Data Acquisition}

\subsection{Test Site}

The field site is located on the Qinghai-Tibet highway, which is close to Tuotuo River, Hoh Xil plateau region, center of Tibetan Plateau, China (N 34 $10^{\prime}$, E $\left.92^{\circ} 23^{\prime}\right)$. The data collection site is on the Qinghai-Tibet Highway, $40 \mathrm{~km}$ south of Tanggula Town (Figure 1). The range of temperature change with the seasons is large [16,17]. Affected by high altitude, the average surface temperature in the area is $10-14^{\circ} \mathrm{C}$ lower than other areas in the same latitude. However, the free temperature in summer is $5-7^{\circ} \mathrm{C}$ higher than the temperature in the same latitude plain. The annual average temperature in the study area is below $0{ }^{\circ} \mathrm{C}$, and the lowest is $-7.0^{\circ} \mathrm{C}$. The average temperature in June-September is positive during the year, and the temperature is negative from October to May. The average temperature in July is the highest, about $5.0-6.0^{\circ} \mathrm{C}$, and the lowest in January is about -20.0 to $-16.0{ }^{\circ} \mathrm{C}$ [7]. In addition, road construction in permafrost areas affects the thermal regime of frozen soils which results in permafrost degradation and road damage [18]. Due to long-term man-made construction activities and vehicle traffic, the seasonal thawing permafrost on the Qinghai-Tibet Highway is very 
obvious. Due to the heterogeneity of the permafrost medium on the Qinghai-Tibet Highway, seasonal ablation caused many road collapses and undulations [7].

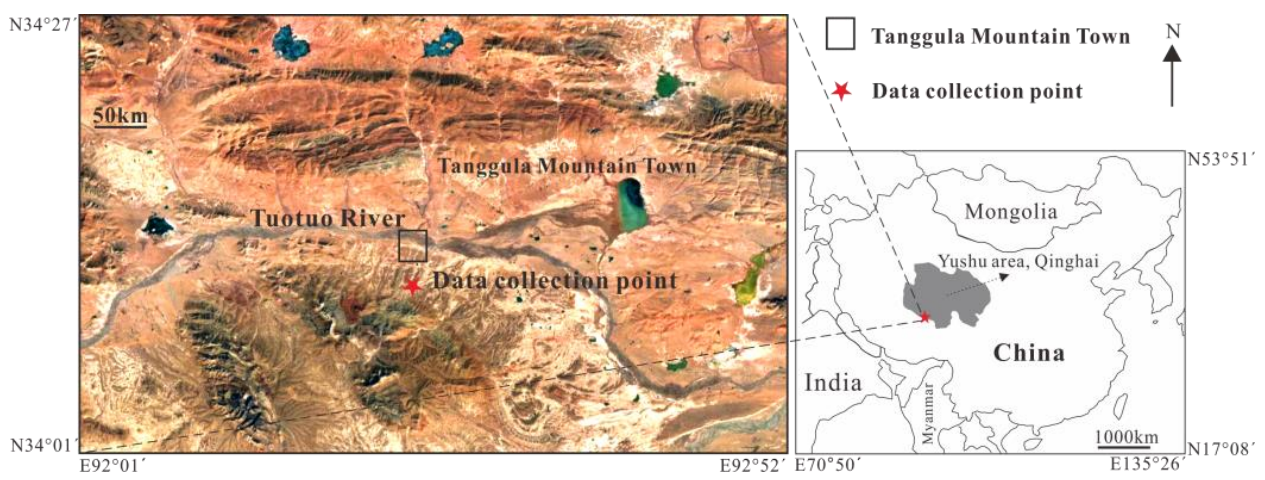

Figure 1. Location map of test site.

\subsection{Test Experiment and Data Acquisition}

The SIR-20 GPR (GSSI, Inc.) system equipped with $200 \mathrm{MHz}$ central frequency antennas was used to perform wheel survey acquisition in the test site. The measurement line layout is shown in Figure 2. Lines 1 to 6 are arranged along the direction of the road, with a line spacing of 3 meters; lines 7 to 12 are arranged perpendicular to the road, with a line spacing of 2 meters. Due to the width of the road, line 1 to 6 have 181 traces, line 7 to 12 have 101 traces. The time window of each trace is $200 \mathrm{~ns}$ with 512 samples. Using the measuring wheel, we use the zero offset distance measurement mode. Figure 3 shows the spatial distribution of 12 lines of data.
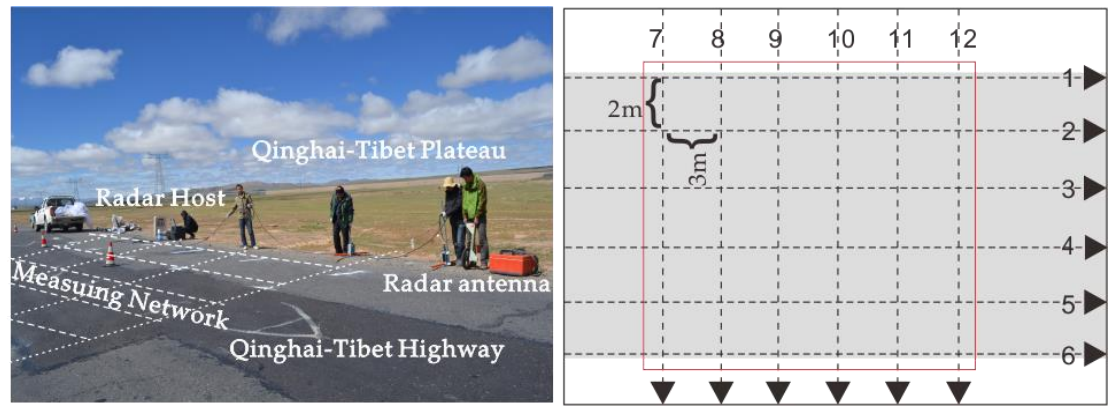

Figure 2. Test experiment and measurement line layout.

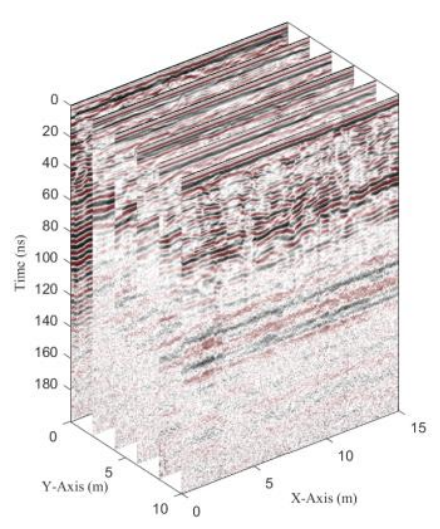

(a)

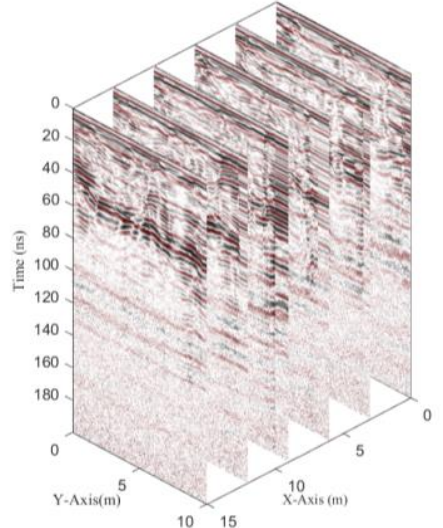

(b)

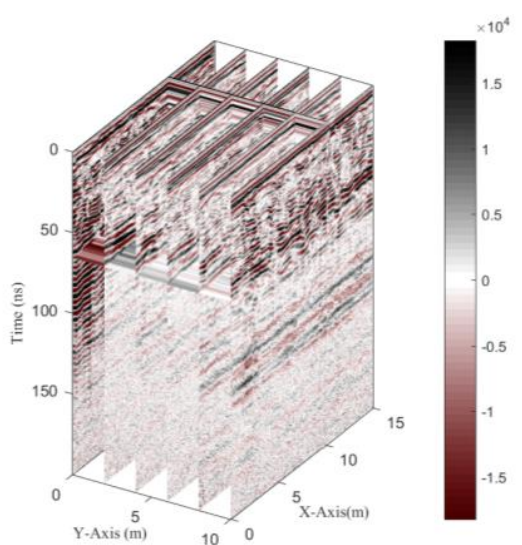

(c)

Figure 3. The spatial distribution of 12 lines of data. (a) Lines 1,2,3,4,5,6; (b) lines 7,8,9,10,11,12; (c) Cross and slice display. 


\subsection{Experimental Data Processing}

In order to ensure the quality of the data, we performed spectrum analysis and imaging capability analysis on these 12 GPR data. The data is centered at $200 \mathrm{MHz}$. In the frequency range from 0 to $500 \mathrm{MHz}$, the data energy is strong, ensuring the spectral integrity of its signal. The original data collected by the experiment uses a distance sampling mode in which the shielded antenna approximates zero offset. The amplitude data of the original data is distributed between $0-800 \mathrm{MHz}$ and carries background noise pollution (Figure 4a). After noise suppression, the amplitude energy is mainly concentrated in the $50-400 \mathrm{MHz}$ interval. This result is consistent with the signal characteristics of the antenna with a center frequency of $200 \mathrm{MHz}$ (Figure $4 \mathrm{~b}$ ). In addition, due to the small influence from external noise and terrain interference, in order to better serve GPR data interpretation, the processing steps can be summarized as follows: correct position, background removal, frequency filtering, horizontal scaling and deconvolution.
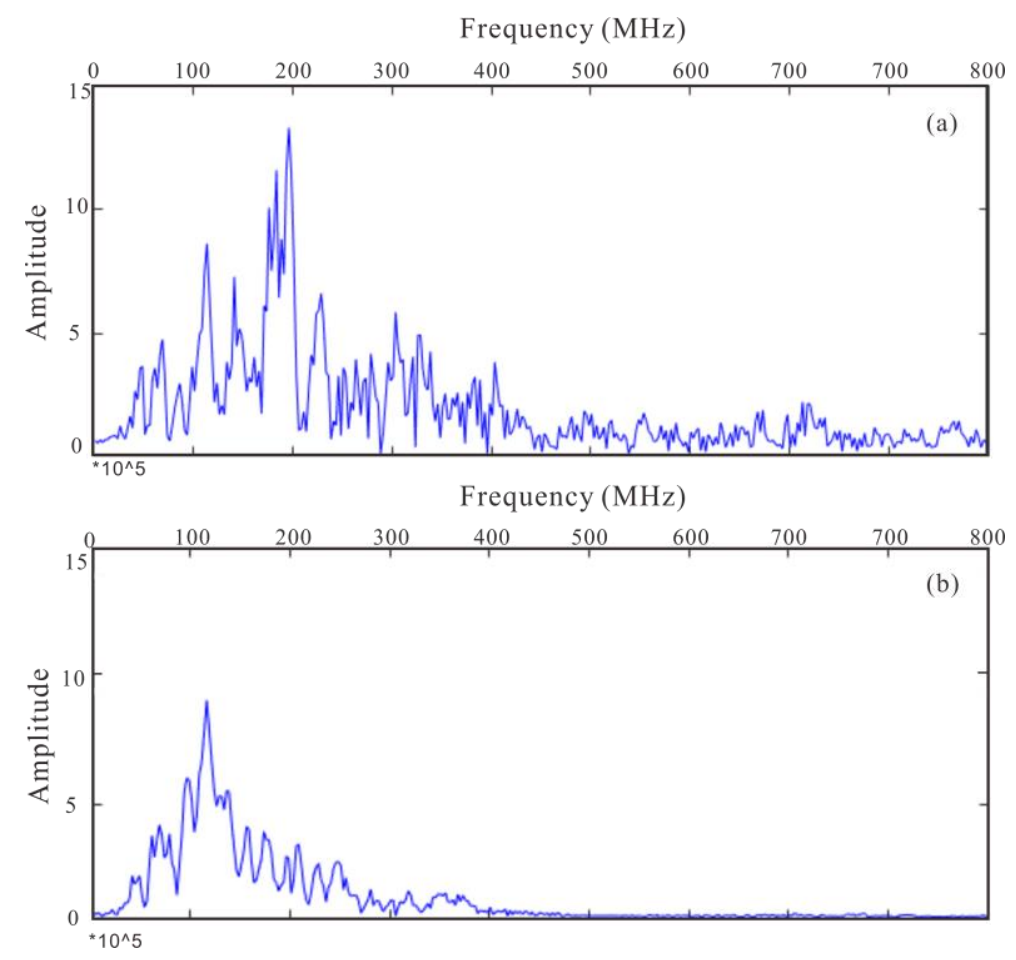

Figure 4. Data spectrum analysis. (a) Raw data; (b) processed data.

Figure 5 is a lithology and stratigraphic column of the shoulder-drilled formation in the data acquisition block. The surface layer is artificial gravel and soil filling with a thickness of $1 \mathrm{~m}$. When the depth is within 7 meters, the formation lithology is mainly clay and sandstone. The lithological changes within 7 meters are mainly reflected in the size of the grain. In formations above $3.7 \mathrm{~m}$ depth, the melting of the frozen soil results in water in the medium. When the depth is greater than 3.7, the water content in the frozen soil is extremely low, and the water is stored in the soil in the form of ice. 


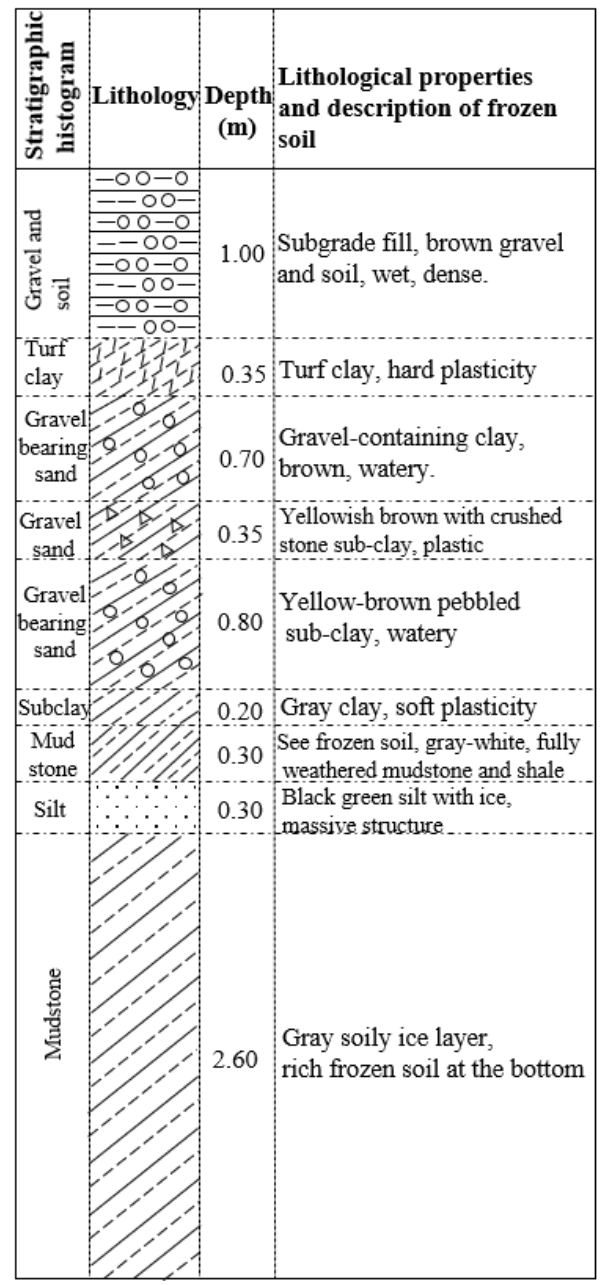

Figure 5. Lithology and stratigraphic column.

\section{Data Analysis and Discussion}

\subsection{Frozen Soil Layered Description}

The continuity of the GPR data in phase and the strong impedance interface are often used to divide the various interfaces of the frozen soil [19]. Figure 6 shows amplitude of the 2 and 5 line which are parallel to the road's alignment and the 8 and 12 lines which are perpendicular to the road alignment. Due to the change of the dielectric constant, a relatively reflective interface appears in the vicinity of $20 \mathrm{~ns}, 50 \mathrm{~ns}$ and $100 \mathrm{~ns}$ of the data.

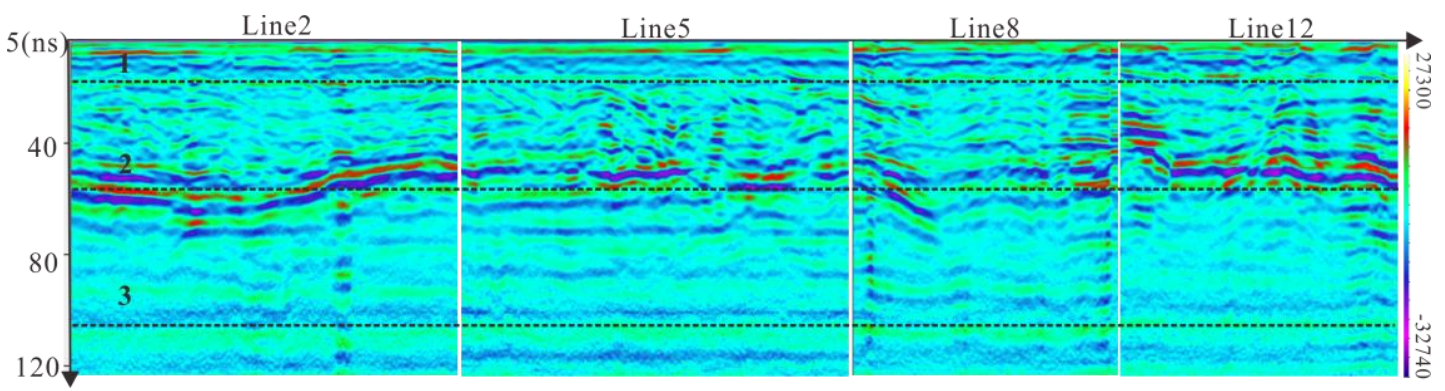

Figure 6. Amplitude of ground penetrating radar (GPR) data: the black dashed lines 1, 2, and 3 respectively notes $20 \mathrm{~ns}, 50 \mathrm{~ns}$ and $100 \mathrm{~ns}$. 
In order to more accurately determine the location of the interface, we performed waveform view and attenuation attributes analysis (Figure 7) on the data. In Figure 7, the attenuation attributes of the GPR signal are calculated. That represents the instantaneous power of electromagnetic waves over time in different media [20]. Mean and median attenuation respectively represent two calculation methods. In the calculation process, they use the mean and median instantaneous amplitude of all traces. The mean is the arithmetic average of a set of numbers, or distribution. It is the most commonly used measure of central tendency of a set of numbers. The median is described as the numeric value separating the higher half of a sample, a population, or a probability distribution, from the lower half. Two statistical algorithms can be used to avoid the incompleteness of any one algorithm, making the results more credible. This also determines best fitting models for power-law and exponential attenuation based on the median attenuation data. Among the four physical layers, the attenuation of electromagnetic waves also exhibits differences due to various values in dielectric constant, density, and water content. At time in $20 \mathrm{~ns}, 50 \mathrm{~ns}$, and $100 \mathrm{~ns}$, there is a significant inflection point in the instantaneous power.

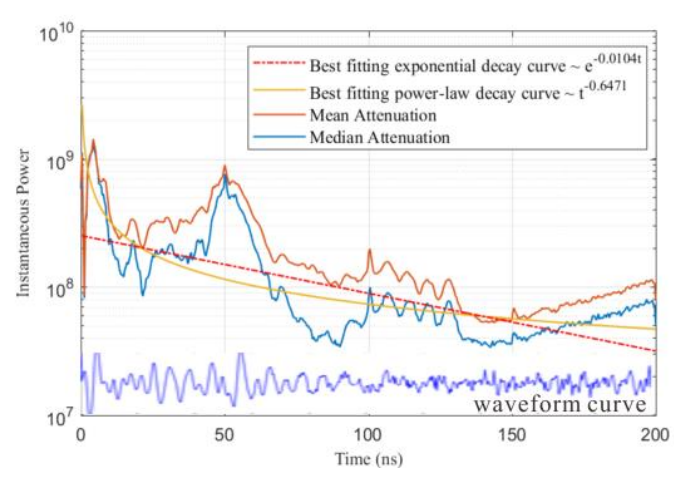

(a)

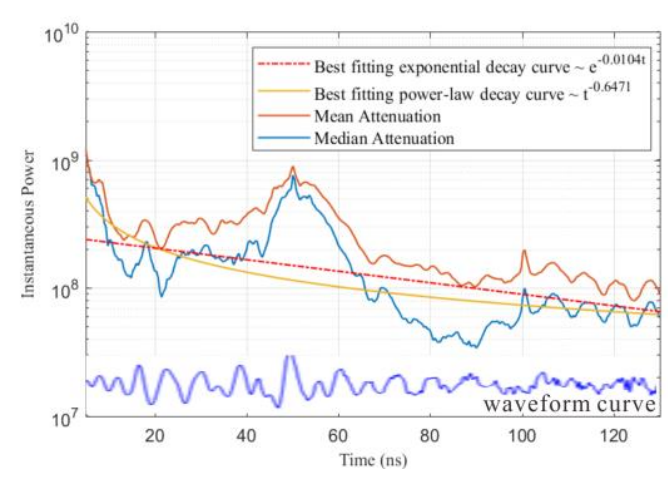

(b)

Figure 7. Electromagnetic waveform view and wave attenuation analysis. (a) Raw data; (b) processed data.

For calculating the attenuation attribute, we use the classic equation as follows:

$$
\beta=20 \times \log _{10} \frac{A_{2}}{A_{1}}
$$

where $A_{2}$ and $A_{1}$ are the amplitude values of two adjacent peaks. A change in the dielectric constant will produce impedance of the electromagnetic wave. Relative to the amplitude attribute, it can better reflect the changing characteristics of the interface. Due to the lack of accurate dielectric constant and physical property information of the buried media, it is difficult to establish the initial model needed to construct absolute wave impedance conversion. Fortunately, relative wave impedance conversion (RAI) does not rely on an initial model. Relative wave impedance is a simplified conversion. It provides a quick and easy way to view data without subjecting it to an conversion process. It highlights areas where there are changes in reflectivity illuminating specific subsurface medium properties [21]. Due to its ability to identify thin layers, it is ideal for radar data interpretation that requires high resolution. The magnitude of reflection coefficient $\gamma_{i}$ indicates the intensity of the reflection between two thawing layers according to the classic Equation (2) [22]:

$$
\gamma_{i}=\frac{\sqrt{\varepsilon_{i}}-\sqrt{\varepsilon_{i+1}}}{\sqrt{\varepsilon_{i}}+\sqrt{\varepsilon_{i+1}}}
$$


where $\varepsilon_{i}$ and $\varepsilon_{i+1}$ are the dielectric constant of permafrost thawing layers $i$ and $i+1$ respectively. Continue to simplify and derive Equation (3):

$$
\lambda=\int \gamma d t \approx \frac{1}{2} \int \frac{\Delta \varepsilon}{2 \varepsilon}=\frac{1}{2} \ln \varepsilon
$$

where $\varepsilon$ is the average dielectric constant value of $\varepsilon_{i}$ and $\varepsilon_{i+1} . \Delta \varepsilon$ is the difference between $\varepsilon_{i}$ and $\varepsilon_{i+1}$. $\lambda$ is relative wave impedance. It is proportional to the natural logarithm of the permittivity values.

Figure 8 shows the relative wave impedance conversion profiles for lines $2,5,8$, and 12 , respectively. Relative to the amplitude attribute, the relative wave impedance data is clearer and the resolution is higher. The black dotted lines 1, 2, and 3 respectively show the stratified positions of the frozen soil physical interface.

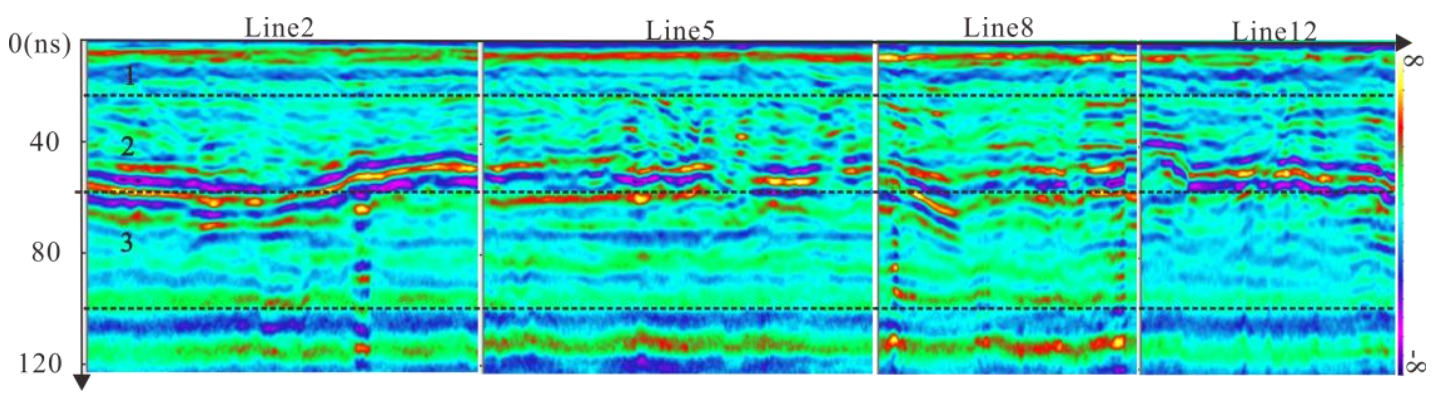

Figure 8. Relative wave impedance conversion profiles.

Based on the above analysis, we divide the seasonal permafrost into four parts. The $0-20 \mathrm{~ns}$ interval is the complete thawing layer; the $20-50 \mathrm{~ns}$ is the thawing layer, wherein the $50 \mathrm{~ns}$ depth is the upper interface of the seasonal permafrost ablation; the $50 \mathrm{~ns}$ is the frozen soil unablated layer, of which $50-100 \mathrm{~ns}$ is the partial ablation of the early frozen soil. The dielectric property change layer, below $100 \mathrm{~ns}$ is a completely frozen soil layer. The reason for the early partial ablation at 50-100 ns was that the depth of $50 \mathrm{~ns}$ was the surface before the Qinghai-Tibet Highway was built. Combined with the drilling measurement data, when the dielectric constant of the medium is 11 , the GPR data has the best correspondence with the formation horizon.

\subsection{Relative Water Content and Temperature Calculation}

The water content calculation of seasonal permafrost is an important part of ground penetrating radar data. Temperature is the main factor affecting the water content of frozen soil. At the same time, due to the change of water content, the physical property parameter information will be directly reflected in the electromagnetic wave data of ground penetrating radar. By mining the multi-attribute information of GPR data, the correspondence between the GPR data attribute and the measured water content and temperature is the basis for the water content calculation using GPR data. When the correlation between the attribute value and the water content is more than $80 \%$, it is judged as a valuable attribute for calculating the water content. By calculation, we get the valuable attribute: The absolute instantaneous amplitude and the weighted average frequency attribute. The weighted average frequency is an improvement to the instantaneous frequency. Instantaneous frequency $w(t)$ is defined as the derivative of the phase of the signal. Signal $z(t)$ can be written as the sum of $N$ items of the index of signal:

$$
z(t)=\sum_{n=0}^{N=+\infty} a_{n}(t) e^{j \phi_{n}(t)}=a(t) e^{j \phi(t)}
$$


where $a_{n}(t)$ is a constant parameter. Because instantaneous frequency is prone to generating spikes and noise for GPR data. To solve this problem, weighted instantaneous frequency is introduced. Weighted instantaneous frequency $\omega(t)$ is defined as follows:

$$
\omega(t)=\frac{\sum_{n=0}^{N=+\infty} a_{n}^{2}(t) \phi_{n}^{\prime}(t)}{\sum_{n=0}^{N=+\infty} a_{n}^{2}(t)}
$$

The weighted average frequency attribute is rarely affected by short wavelength and noise [23]. In Figure 9a,b, there is a high correlation between absolute instantaneous amplitude $(A i)$, the weighted average frequency attribute $\omega$ and the measured water content. The high red absolute instantaneous amplitude and weighted average frequency attribute corresponds well to the water content of the black display. On the mining of valuable attributes representing temperature, we use the correlation value greater than $65 \%$ as the reference value for attribute mining. Due to differences in media properties and particles in the surface roadbed, the correlation between temperature and water content in the surface layer is not high. The weighted average frequency attribute can basically reflect the temperature distribution characteristics of a fully melted layer with water and an incompletely melted ice layer. However, because the temperature change value is not large, the accuracy cannot reach the standard of water content correlation. So, we use this attribute to show the trend of temperature (Figure 9b). It is mainly used as a control factor for the calculation of water content. Figure 10 shows the weighted average frequency attribute reflecting the formation of temperature. Figure 10 indicates the reflecting effect of temperature in the water-ice contact. The temperature of the fully melted layer is higher than $0{ }^{\circ} \mathrm{C}$ and the temperature of the ice-containing layer is lower than $0{ }^{\circ} \mathrm{C}$.

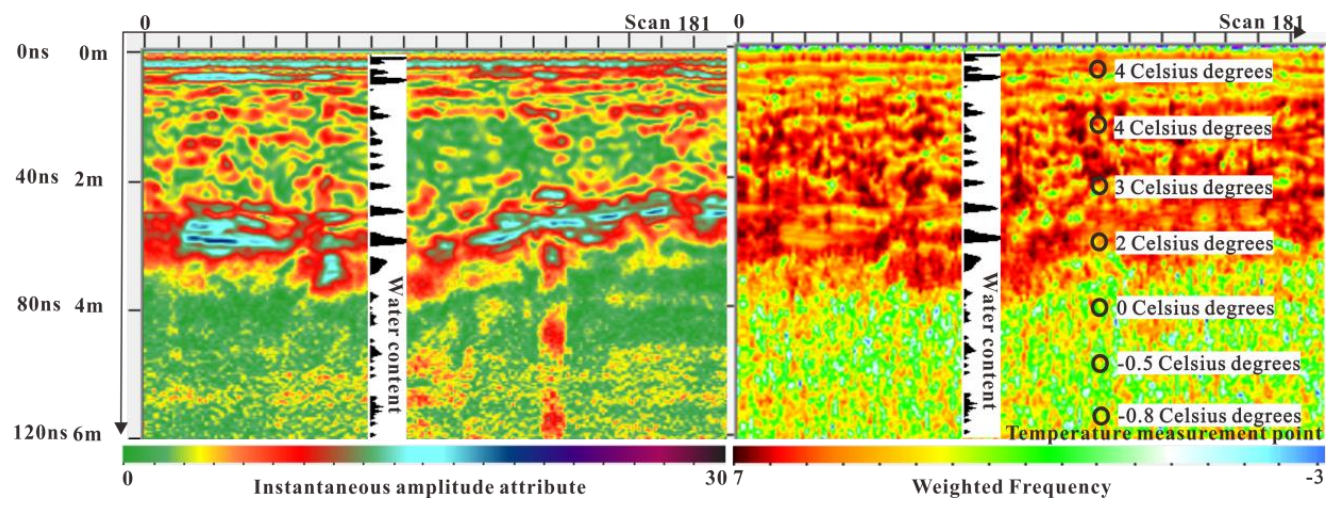

(a)

(b)

Figure 9. Correspondence diagram between electromagnetic wave attribute and measured data. (a) Absolute instantaneous amplitude attribute; (b) weighted average frequency attribute; water content is inserted in (a) and (b); black circle notes temperature in (b). The dielectric constant of the medium is 11.

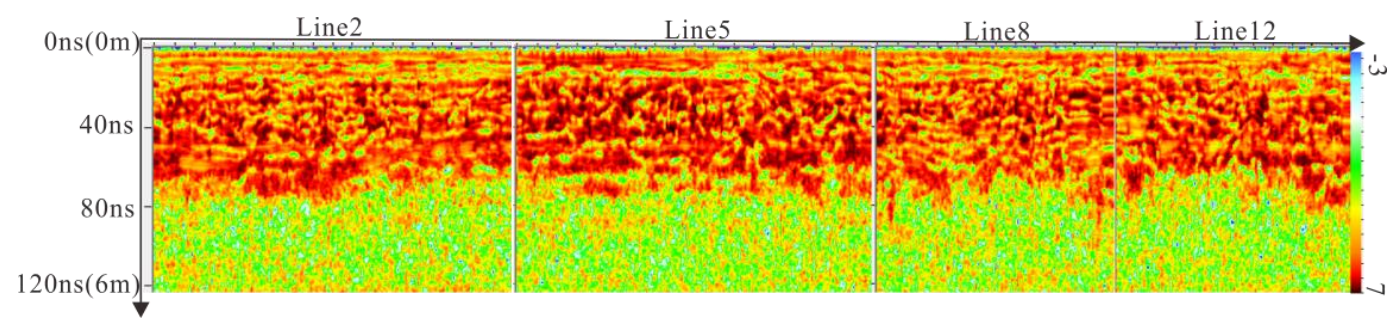

Figure 10. The weighted average frequency attribute. 
The relationship between measured data and electromagnetic wave attributes mining and screening studies provides attenuation attribute, relative wave impedance, absolute instantaneous amplitude attribute and the weighted average frequency attribute for water content conversion. Values of water content in the medium lead to differences in dielectric constant. The change of dielectric constant of the medium causes the change of the electromagnetic wave impedance value. Because of the positive correlation among them, the electromagnetic wave impedance parameter can be added to the calculation of the relative water content. The water content calculation operator can be expressed as:

$$
\begin{aligned}
& \delta=\frac{\beta+\lambda+A i+\omega}{4} \\
& \beta=20 \times \log _{10} \frac{A_{2}}{A_{1}} \\
& \lambda=\int \gamma d t=\frac{1}{2} \ln \varepsilon
\end{aligned}
$$

where attenuation attribute $\beta$, relative wave impedance $\lambda$, absolute instantaneous amplitude attribute $(A i)$ and the weighted average frequency attribute $\omega$ are the normalized values parameters. $\delta$ is water content conversion operator. The Topp model is a formula for accurately calculating the relationship between soil water content and dielectric constant. Since GPR data cannot directly obtain accurate absolute dielectric constants, substitute Equation 4 into the Topp model formula [24]:

$$
\theta=-0.53 \times 10^{-2}+2.92 \times 10^{-2} \delta-5.5 \times 10^{-4} \delta^{2}+4.3 \times 10^{-6} \delta^{3}
$$

where $\theta$ is relative water content. Figure 12 shows the working methods and processes of the entire research work. The relative water content information is mined from the GPR data by calculating the value of the sensitive attribute and the relative wave impedance (Figure 11).

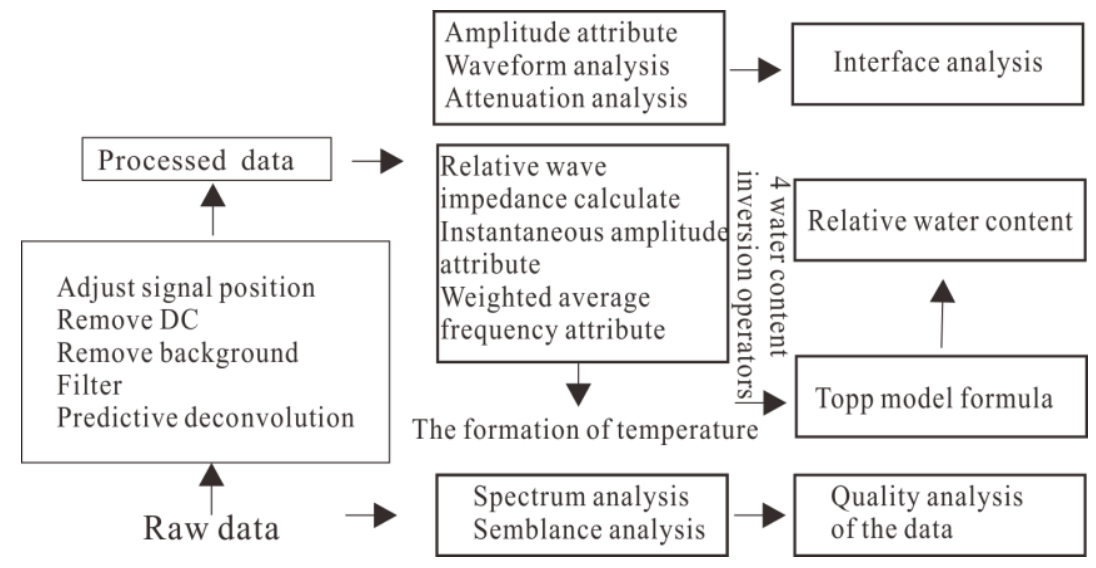

Figure 11. Method flow diagram.

We use Equation 5 for the calculation of relative water content. Figure 12 shows the water content profile for the conversion of different line data. Because in the research of frozen soil water content calculation, the main concern area is the complete melting zone of frozen soil nodule between 20 55 $( \pm 10)$ ns. The measured sample data is in good agreement with the water content value calculated by the GPR data in this region. In the $50( \pm 10) \sim 100 \mathrm{~ns}$ and 100-120 ns areas, it is not the area of concern. The soil in this area mainly contains ice, and the measured water content is inferior to the calculated value. In addition, due to the low quality of deep data, deep data carries less physical information. We calculate and present the data within 65 ns. Figure 13 shows the planar variation characteristics of water content at different depths of time. Red represents high water content and green represents low water content. Before $10 \mathrm{~ns}$, due to the roadbed medium, the surface medium was relatively dry and the water content was very low. As the depth increases, the water content in the thawing layer increases. As the depth continues to increase, the water content becomes smaller. These characteristics and values are consistent with the characteristics and measured data of seasonal permafrost ablation. 


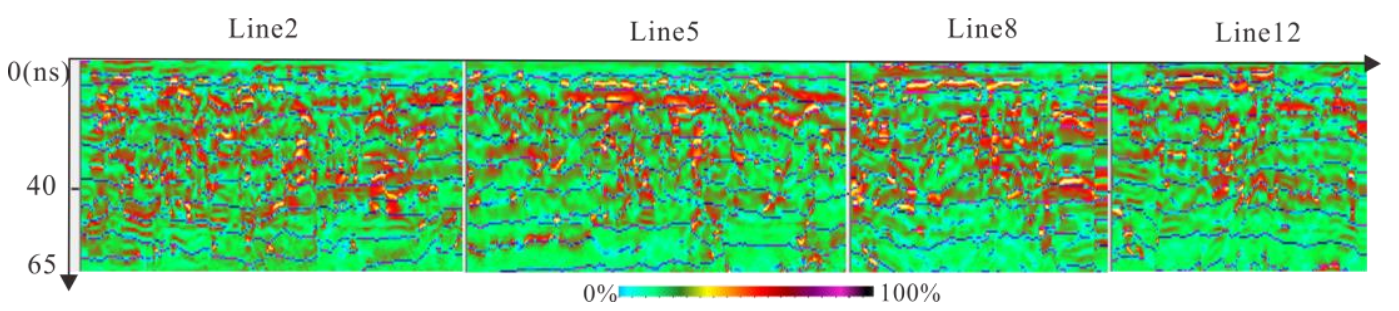

Figure 12. Relative water content calculation for the set of lines 2, 5, 8 and 12.

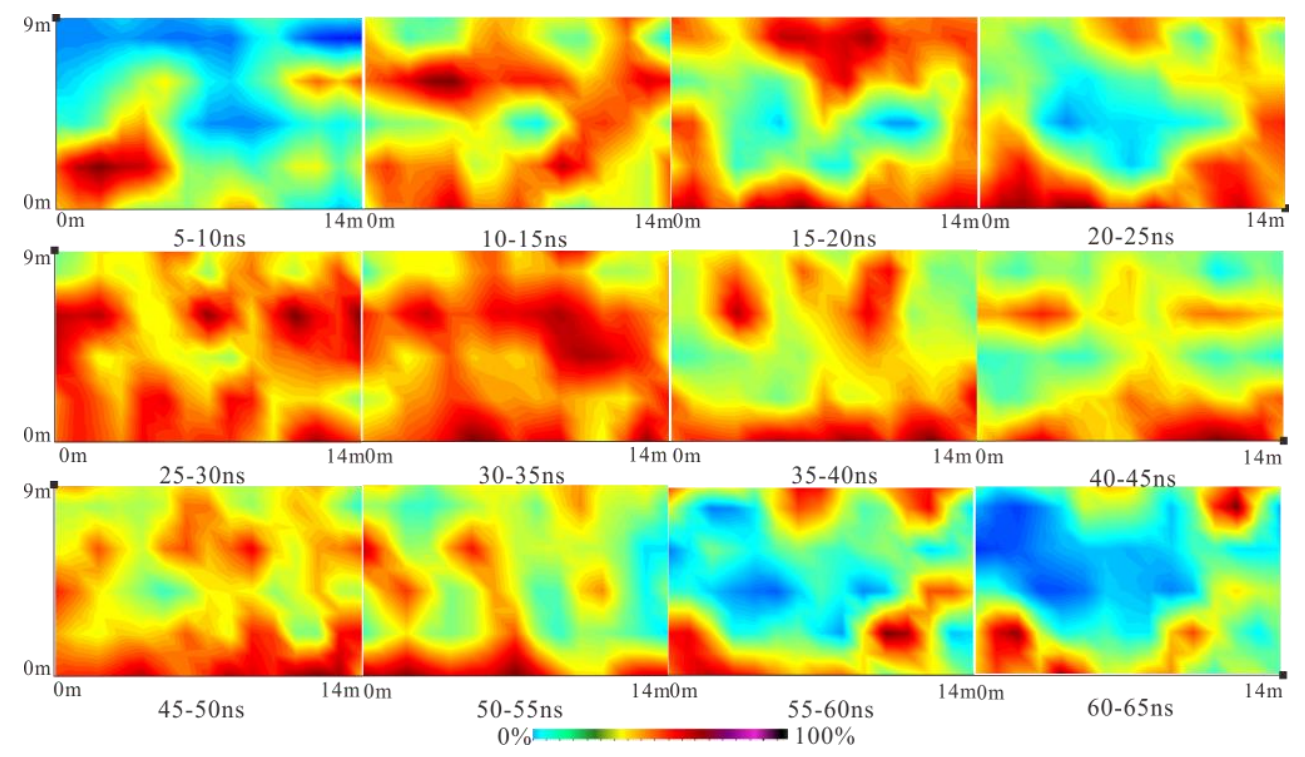

Figure 13. The planar variation characteristics of relative water content at different depths of time.

\section{Conclusions}

We provide an interface identification method for using ground penetrating radar data to carry out seasonal permafrost areas on the Tibetan Plateau. By contrast with the traditional low-resolution method, only the amplitude data change is used to distinguish the interface. In this study, amplitude analysis, the waveform analysis and electromagnetic wave attenuation attribute are used to make a more detailed imaging division of the seasonal permafrost area. The medium in the seasonal permafrost area is divided into a finer four-layer structure. They are: subgrade, frozen soil ablation layer, frozen soil semi-ablative layer and permafrost layer. Through the calibration of the drilling data, dielectric constant of time-depth conversion in the Qinghai-Tibet highway, which is close to Tuotuo River, and the Hoh Xil plateau region, the center of Tibetan Plateau, is near 11.

In addition, when the correlation between the attribute value and the water content is more than $80 \%$, it is judged as a valuable attribute for calculating the water content. Absolute instantaneous amplitude and the weighted average frequency attribute can react well to changes in water content. Using GPR data is more difficult for temperature calculation. The weighted average frequency attribute can basically reflect temperature and change, but its accuracy does not reach the standard of water content correlation.

Since the GPR data cannot directly obtain the dielectric constant throughout the medium, we replace the dielectric constant with a calculation factor that combines the attenuation attribute, relative wave impedance attribute, absolute instantaneous amplitude attribute, and the weighted average frequency attribute to calculate the relative moisture content. The calculation results are in good agreement with the actual sample measurements in the frozen soil ablation zone. When the ice-containing state occurs in the soil, the error of this calculation method becomes large. 
Author Contributions: Q.W. and Y.S. jointly collected data needed for research on the Qinghai-Tibet Plateau. Q.W. studied data processing algorithms; Y.S. studied frozen soil theory and results verification. They completed the research and article together.

Funding: This was financially supported by National 973 Project of China (NO.2012CB026104), the National Natural Science Foundation (NSFC) under grants (No.51578053, No. 41772330 and No. 41171064), Beijing Information Science and Technology University Research Fund project (182501), the State Key Laboratory of Frozen Soils Engineering(Grant Nos.SKLFSE201808), the Scientific Research Project of Beijing Educational Committee (KM201811232010), Beijing Science and Technology Innovation Service Capacity BuildingFundamental Research Fund(PXM2018_014224_000032) and the Project for Promoting Connotative Development of Universities-"Information +" project.

Acknowledgments: The authors would like to thank the professors of Beijing Jiaotong University for suggestions on the theory of seasonal permafrost background. The authors would like to thank to the National 973 Project of China for support. The authors are also grateful to the reviewers and editor for their valuable comments and remarks.

Conflicts of Interest: The authors declare no conflict of interest. The funders had no role in the design of the study; in the collection, analyses, or interpretation of data; in the writing of the manuscript; or in the decision to publish the results.

\section{References}

1. Zhou, Y.W.; Guo, D.X.; Qiu, G.Q. Geocryology in China; Beijing Science Press: Beijing, China, 2000; pp. 5-35.

2. Niu, F.J.; Lin, Z.H.; Liu, H.; Xu, Z.Y. Characteristics of Roadbed Settlement in Embankment-Bridge Transition Section along the Qinghai-Tibet Railway in Permafrost Regions. Cold Reg. Sci. Technol. 2001, 65, 437-445. [CrossRef]

3. Wang, S.L. Discussion on the Degradation of Frozen Soil and the Problem of Frozen Soil Environment in the Qinghai-Tibet Plateau. In Proceedings of the Fifth National Glacier and Frozen Soil Conference, Lanzhou, China, 1996; pp. 11-17.

4. Wu, Q.B.; Dong, X.F.; Liu, Y.Z. Response Analysis of Permafrost along the Qinghai-Tibet Highway to Climate Change and Engineering Impact. Glacier 2005, 27, 50-54.

5. Jin, H.J.; Zhao, L.; Wang, S.L.; Jin, R. Characteristics and Degradation Modes of Frozen Soil Along the Qinghai-Tibet Highway. Chin. Sci. Bull. D Earth Sci. 2006, 11, 1009-1019.

6. Davis, J.L.; Annan, A.P. Ground Penetrating Radar for High Resolution Mapping of Soil and Rock Stratigraphy. Geophys. Prospect. 1989, 37, 531-551. [CrossRef]

7. Luo, J. Study on instability and susceptibility evaluation of frozen soil slope in Qinghai-Tibet Engineering Corrido. PhD Thesis, Chinese Academy of Sciences, Lanzhou, China, 2015.

8. Arcone, S.A.; Lawson, D.E.; Delaney, A.J. Ground-Penetrating Radar Reflection Profiling of Groundwater and Bedrock in An Area of Discontinuous Permafrost. Geophysics 1998, 63, 1573-1584. [CrossRef]

9. Arcone, S.A.; Prentice, M.L.; Delaney, A.J. Stratigraphic Profiling with Ground-Penetrating Radar in Permafrost: A Review of Possible Analogs for Mars. J. Geophys. Res. 2002, 107, 1-14. [CrossRef]

10. Xiao, J.; Liu, L. Signal Fusion Using Extrapolation with Deterministic Deconvolution on Multi-Frequency Qinghai-Tibet Railway GPR Data for Permafrost Subgrade Detection. In Proceedings of the 15th International Conference On Ground Penetrating, Radar, Brussels, Belgium, 30 June-4 July 2014; pp. 586-589.

11. Robert, C. Time-Domain Reflectometry Method and Its Application for Measuring Moisture Content in Po Rous. Mater. Meas. 2009, 42, 329-336.

12. Weiler, K.W.; Teenhuis, T.S.; Kung, K.S. Comparison of Ground Penetrating Radar and Time Domain Reflectometry as Soil Water Sensors. Soil Sci. 1998, 62, 1237-1239. [CrossRef]

13. Grote, K.H.; Ubbard, S.; Rubin, Y. Field -Scale Estimation of Volumetric Water Content Using Ground Penetrating Radar Ground Wave Techniques. Water Resour. Res. 2003, 39, 1-13. [CrossRef]

14. Huisman, J.A.; Sperl, C.; Bouten, W. Soil Water Content Measurements at Different Scales: Accuracy of Time Domain Reflectometry and Ground Penetrating Radar. J. Hydrol. 2001, 245, 48-58. [CrossRef]

15. Shen, Y.P.; Zuo, R.; Liu, J.K. Characterization and Evaluation of Permafrost Thawing Using GPR Attributes in The Qinghai-Tibet Plateau. Cold Reg. Sci. Technol. 2018, 151, 302-313. [CrossRef]

16. Zhao, L.; Cheng, G.; Li, S. Thawing and Freezing Processes of Active Layer in Wudaoliang Region of Tibetan Plateau. Chin. Sci. Bull. 2000, 45, 2181-2186. [CrossRef] 
17. Zhao, W.K.; Forte, E.; Pipan, M.; Tian, G. Ground Penetrating Radar (GPR) Attribute Analysis for Archaeological Prospection. J. Appl. Geophys. 2014, 97, 107-117. [CrossRef]

18. Hinkel, K.M.; Doolittle, J.A.; Bockheim, J.G.; Nelson, F.E.; Paetzold, R.; Kimble, J.M.; Travis, R. Detection of Subsurface Permafrost Features with Ground-Penetrating Radar, Barrow, Alaska. Permafr. Periglac. Process. 2001, 12, 179-190. [CrossRef]

19. Liu, L.B.; Qian, R.Y. Ground Penetrating Radar: A Critical Tool in Near-Surface Geophysics. Chin. J. Geophys. 2015, 58, 2606-2617.

20. Tzanis, A. matGPR Release 2: A Freeware MATLAB Package for The Analysis \& Interpretation of Common and Single Offset GPR Data. Fast Times 2010, 15, 17-43.

21. Rahmani, A.; Belmokhtar, A.; Murineddu, A.; Khazanehdari, J.; English, J.; Roumane, H.; Godfrey, B. The Art of Seismic Conversion-An Example from Erg Chouiref Algeria. SEG Tech. Program Expand. Abstr. 2006, 25, 264-268.

22. Maser, K.R. Ground Penetrating Radar Surveys to Characterize Pavement Layer Thickness Variations at GPS Sites; Report; Strategic Highway Research Program National Research Council: Washington, DC, USA, 1994; p. 397.

23. Loughlin, P.J. Comments on the interpretation of instantaneous frequency. IEEE Signal Process Lett. 1997, 4, 123-125. [CrossRef]

24. Topp, G.C.; Davis, J.L.; Annan, A.P. Electromagnetic Determination of Soil Water Content: Measurements in Coaxial Transmission Lines. Water Resour. Res. 1980, 16, 574-582. [CrossRef]

(C) 2019 by the authors. Licensee MDPI, Basel, Switzerland. This article is an open access article distributed under the terms and conditions of the Creative Commons Attribution (CC BY) license (http://creativecommons.org/licenses/by/4.0/). 